\title{
The response of four calcium hydroxides on monkey pulps
}

\author{
D. R. Heys, R. J. Heys, C. F. COX AND J. K. Avery \\ The University of Michigan, Department of Oral Biology, \\ 5211 Dental Research Institute, Ann Arbor, Michigan, U.S.A.
}

\begin{abstract}
Dentinal bridge formation and pulpal responses of four calcium hydroxide materials, pulp capping medicaments, MPC $\circledast$, Experimental MPC-12, Dycal ${ }^{\circledR}$ and Pulpdent ${ }^{\circledR}$, were evaluated in primary and permanent monkey teeth. A total of 60 primary and 60 permanent teeth were used with each material placed in a Class V cavity exposure in Rhesus monkey teeth. The materials were placed on the exposed pulp tissue and were histologically evaluated at 3 days, 5 weeks and 8 weeks. After perfusion the teeth were processed using routine histological procedures. The 3-day pulpal responses in both primary and permanent teeth were moderate, characterized by disruption of the pulpal tissue directly beneath the exposure site and a zone of acute inflammation and hemorrhage in the underlying pulp. The 5-week response showed histological differences between the four medicaments, with Dycal producing the least amount of pulpal irritation with reparative dentin bridges occurring in $50 \%$ of the permanent teeth. Experimental MPC-12 stimulated one reparative dentin bridge, while Pulpdent and MPC showed no evidence of bridge formation. Pulpal responses to Dycal were moderate and moderate to severe for the other calcium hydroxide compounds. No reparative dentin bridges were seen in the primary teeth at 5 weeks with any of the materials, and the pulpal responses were of a moderate degree at that time. Eight-week responses were similar to the 5-week responses. Dycal provoked a slight to moderate pulpal response with $50 \%$ success at bridging. Experimental MPC-12 initiated pulpal responses in the moderate to severe range with some bridging evident. Pulpdent incited moderate to severe histological responses with three teeth demonstrating bridge formation, and MPC provoked severe pulpal responses with no bridging. Primary teeth showed some bridging for all compounds except those treated with MPC, in which no evidence of bridging occurred, and moderate to severe pulpal responses were present.
\end{abstract}

\section{Accepted for publication 21 May 1980}

Managing the exposure of the dental pulp has been something which clinicians face frequently in the course of dental practice. Zander (1939) was among the first to report on the use of a $\mathrm{Ca}(\mathrm{OH})_{2}$ material as a treatment for the healing of the exposed dental pulp, and he speculated that the success of $\mathrm{Ca}(\mathrm{OH})_{2}$ was related to its high alkalinity. Since Zander's study, the marketplace has seen the appearance of many $\mathrm{Ca}(\mathrm{OH})_{2}$ containing materials for use on the dental pulp. Phaneuf et al. (1968) compared three of these com- mercially available $\mathrm{Ca}(\mathrm{OH})_{2}$ compounds (Dycal, Pulpdent, Hydrex) in human primary teeth. They reported radiographic evidence of a radiopaque bridge in tooth pulps 28 days after treatment with Pulpdent. Pulps treated with Dycal exhibited some evidence of bridging; however, pulps treated with Hydrex exhibited no bridging within the span of their study. Their histological data revealed a cellular osteodentin bridge formation in pulps treated 28 days previously with Pulpdent and after 50 days' 
treatment with Dycal. Fibrillar dentin production occurred 54 days after treatment with Pulpdent and 70 days after treaiment with Dycal. Pulps treated with Hydrex showed no apparent attempt at repair or dentinal bridging. Stanley \& Lundy (1972) and Tronstad (1974) reported Dycal to be an effective pulp capping agent.

Zander \& Glass (1949) and Nyborg (1955) have suggested that the success obtained with calcium hydroxide as a pulp capping agent is related to its high alkalinity. Fisher \& McCabe (1978) and Tronstad \& Birkeland (1971) have evaluated the $\mathrm{pH}$ of Dycal to be around 11 and the $\mathrm{pH}$ of Pulpdent near 12. MPC has a $\mathrm{pH}$ near $10^{*}$.

The purpose of this study was to compare the effects of three commercially available $\mathrm{Ca}(\mathrm{OH})_{2}$ compounds and one experimental $\mathrm{Ca}(\mathrm{OH})_{2}$ compound on the pulp exposures of monkey teeth. The $\mathrm{pH}$ of the materials will also be related to the histology of the tissues.

\section{Material and Methods}

Four calcium hydroxide compounds $\left(D_{\text {ycal }}{ }^{* *}\right.$ pH 11, Pulpdent $\dagger$ pH 12, MPC $\ddagger$ pH 10, and Experimental MPC pH 12 [Experimental MPC-12§]) were employed as test materials. These materials were placed on the exposed pulps of Rhesus monkey teeth for periods of 3 days, and 5 and 8 weeks according to the American National Standards Committee MD-156 (1974). A total of 60 permanent and 60 primary teeth were used. This gave five teeth per compound per time period. All

\footnotetext{
* Personal Communication, Kerr Mg. Co.

** L. D. Caulk, Milford, DE. (Batch\# 16969).

†Pulpdent Corporation, Boston, MA. (Batch \#5251).

$\$$ Kerr Manufacturing Company, Romulus, MI. (Batch \#04321057).

$\S$ Kerr Manufacturing Company, Romulus, Mi.
}

materials were randomly distributed in anterior and posterior teeth of both arches. All materials were placed in Class V cavity preparations which were prepared with a \#35 inverted cone bur in the permanent teeth, and a \#33- $\frac{1}{2}$ inverted cone bur in the primary teeth. All cavity preparations were prepared by high speed $(250,000 \mathrm{rpm})$ air turbine with water and air spray. The cavity floor was thinned so that the outline of the pulp could be seen; then a sterile explorer was used to reflect the remaining dentin and expose the pulp. Hemorrhage was controlled with sterile cotton pellets and the entire cavity floor was then covered with the medicament. After the medicament set, the remainder of the cavity preparation was restored with amalgam.

At the time of sacrifice, the monkeys were anesthetized and perfused with $10 \%$ phosphate buffered formalin (PBF) (Cox et al. 1977). The teeth were surgically removed from the jaw, decalcified, embedded in paraffin, serially cut at $10 \mu$, and stained with hematoxylin and eosin.

In an attempt to control bias of data recording, the tissue sections were evaluated by the investigators prior to their knowledge of the material used. Histological responses were categorized into slight, moderate and severe, according to Mjör \& Tronstad (1972).

\section{Results}

The 3-day histological responses to Dycal and MPC (Figs 1 and 2) were similar in both primary and permanent dentitions. The pulpal responses were moderate and characterized by an area of hemorrhage and acute inflammatory cells subjacent to the medicament interface. Pulpal to this zone were vital tissues. Responses to Experimental MPC-12 and Pulpdent were 


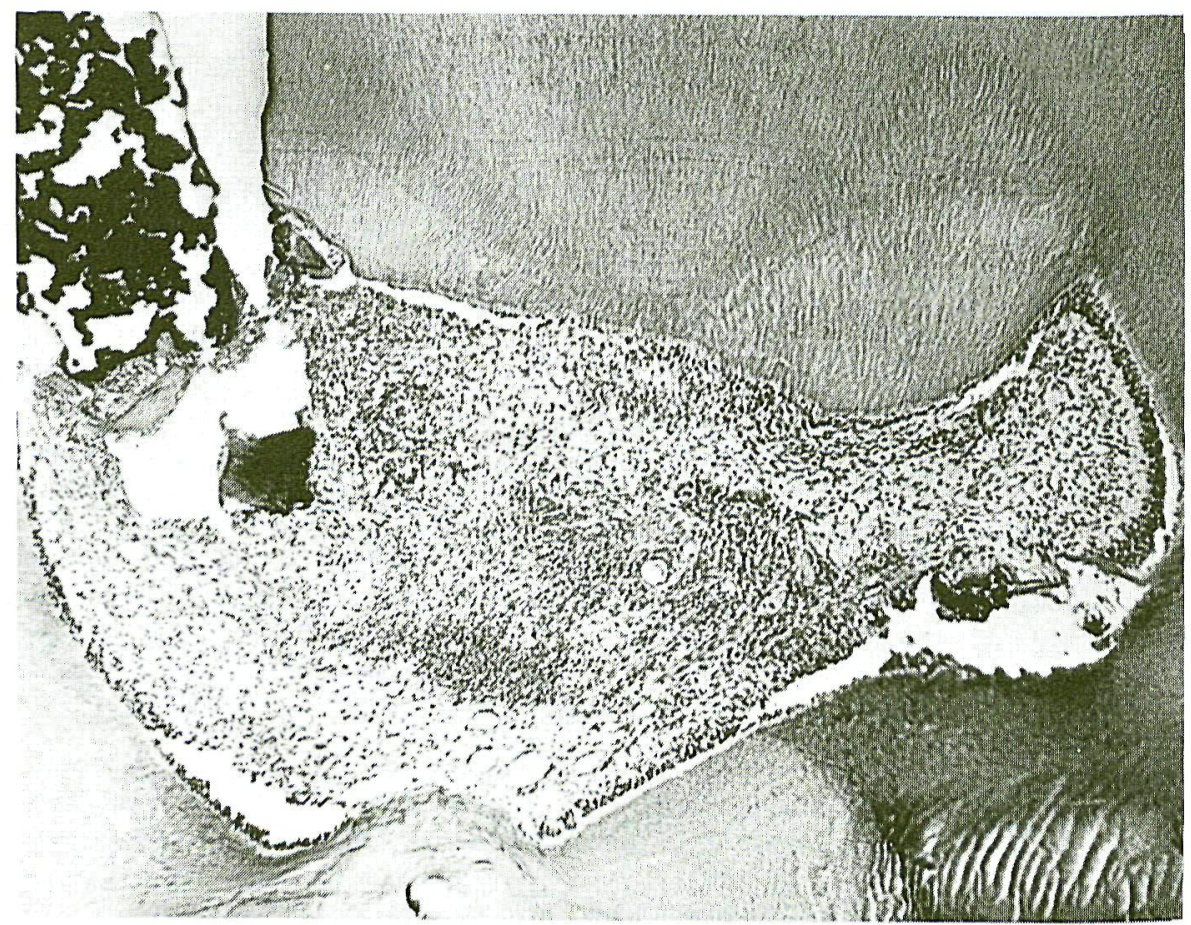

Fig. 1. This is a primary tooth in which MPC was placed 3 days earlier. Some tissue disruption beneath the medicament and a moderate underlying inflammation are observed. Mag. $\times 32$.

similar but differed in the amount of tissue involvement. Some teeth treated with Dycal and MPC had a zone of necrosis beneath the medicament, and some teeth had vital tissue adjacent to the medicament. Some teeth treated with MPC-12 and Pulpdent had large areas of necrosis beneath the medicament.

Differences between the compounds tested were noted after 5 weeks. At this time the pulpal responses of the primary teeth paralleled those seen in the permanent teeth except that a greater percentage of the pulp was involved in primary teeth due to smaller pulps. Responses to Dycal (Fig. 3) were the most favorable at 5 weeks. Dentin bridging was observed in $50 \%$ of the teeth at the medicament interface. The dentin bridge was composed of cellular reparative dentin. The pulpal responses were slight to moderate with some chronic inflammatory cells underlying the bridge and a slight increase in vascularity. In those teeth with no reparative dentin bridge the pulpal response was moderate. In primary teeth all responses were moderate for all compounds, and no evidence of dentin bridging was noted. With Experimental MPC-12, moderate pulpal responses were noted on all teeth that had shown no evidence of reparative dentin bridge formation. One primary and one permanent tooth showed reparative dentin bridge formation occurring at some distance from the medicament interface and loss of the pulpal tissue between the exposure site and the dentin bridge. The pulpal response in 


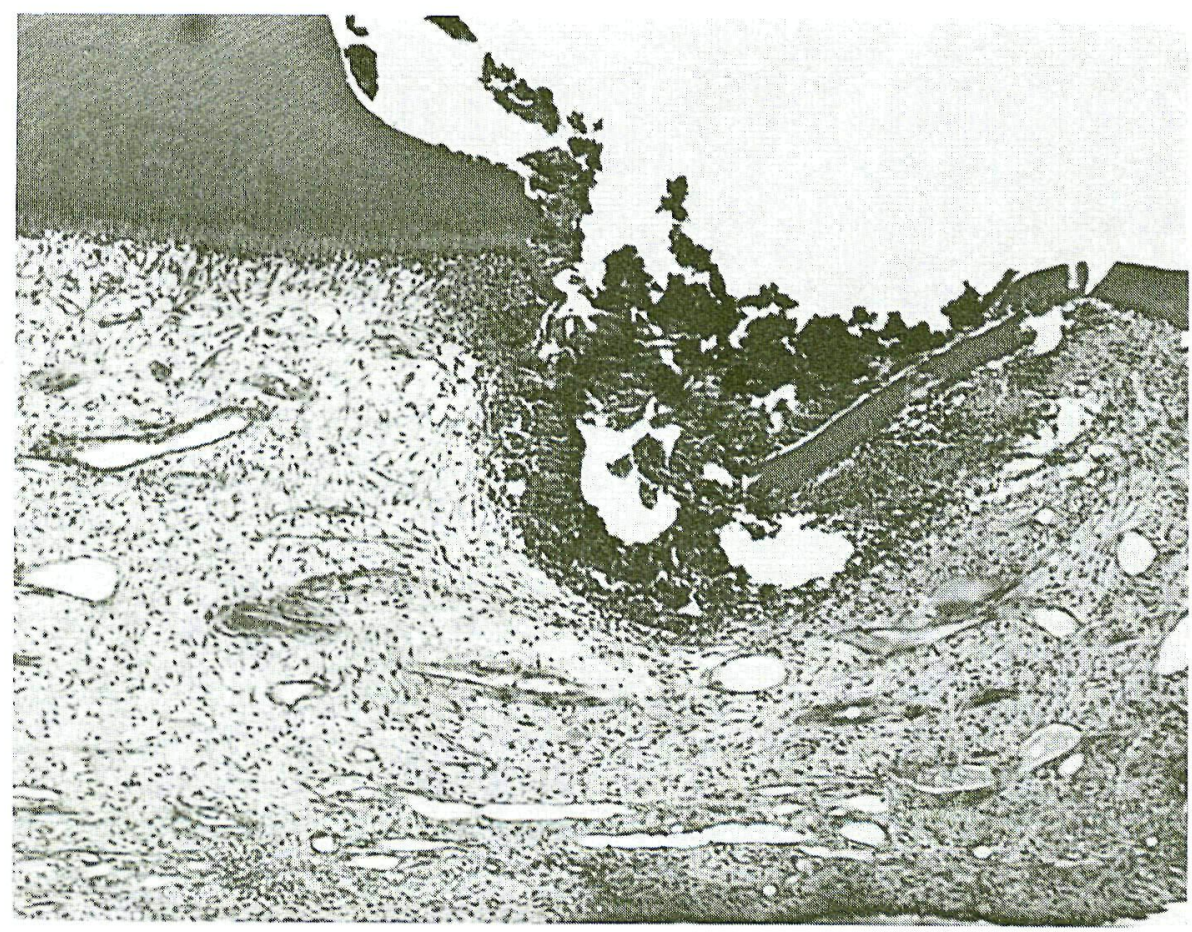

Fig. 2. This is a permanent tooth in which MPC was placed 3 days earlier. It shows a moderate inflammatory response with inflammation and disruption limited to the area beneath the exposure. Mag. $\times 64$.

these teeth was slight to moderate. At this time moderate to severe pulpal responses to MPC and Pulpdent were noted with no reparative dentin bridges observed in either primary or permanent teeth.

Dycal was the most effective reparative dentin bridge potentiator in this study. Eight weeks after Dycal application $50 \%$ of the permanent teeth had a reparative dentin bridge with a slight pulpal response, and moderate inflammation was noted in those teeth where no bridge was evident. A reparative dentin bridge was noted in one primary tooth pulp with a slight to moderate response, whereas four teeth showed no bridge formation with a moderate to severe inflammatory response. Two of five permanent teeth treated with Experimental MPC-12 (Fig. 4) showed reparative dentin bridge formation. These had a slight to moderate pulpal response, whereas in those teeth with no bridges a severe pulpal response was seen. The same was observed in the primary teeth where two reparative dentin bridges were found with pulpal responses slight to moderate. In those primary teeth with no bridges a severe pulpal response was noted involving the majority of the pulp. After 8 weeks one tooth treated with Pulpdent had a dentin bridge and a slight pulpal response (Fig. 5). The other four teeth had pulpal responses ranging from moderate to severe. In the primary teeth in which Pulpdent was placed, one partial reparative dentin bridge was observed with a moderate pulpal response, while four teeth had a severe pulpal response. Both 


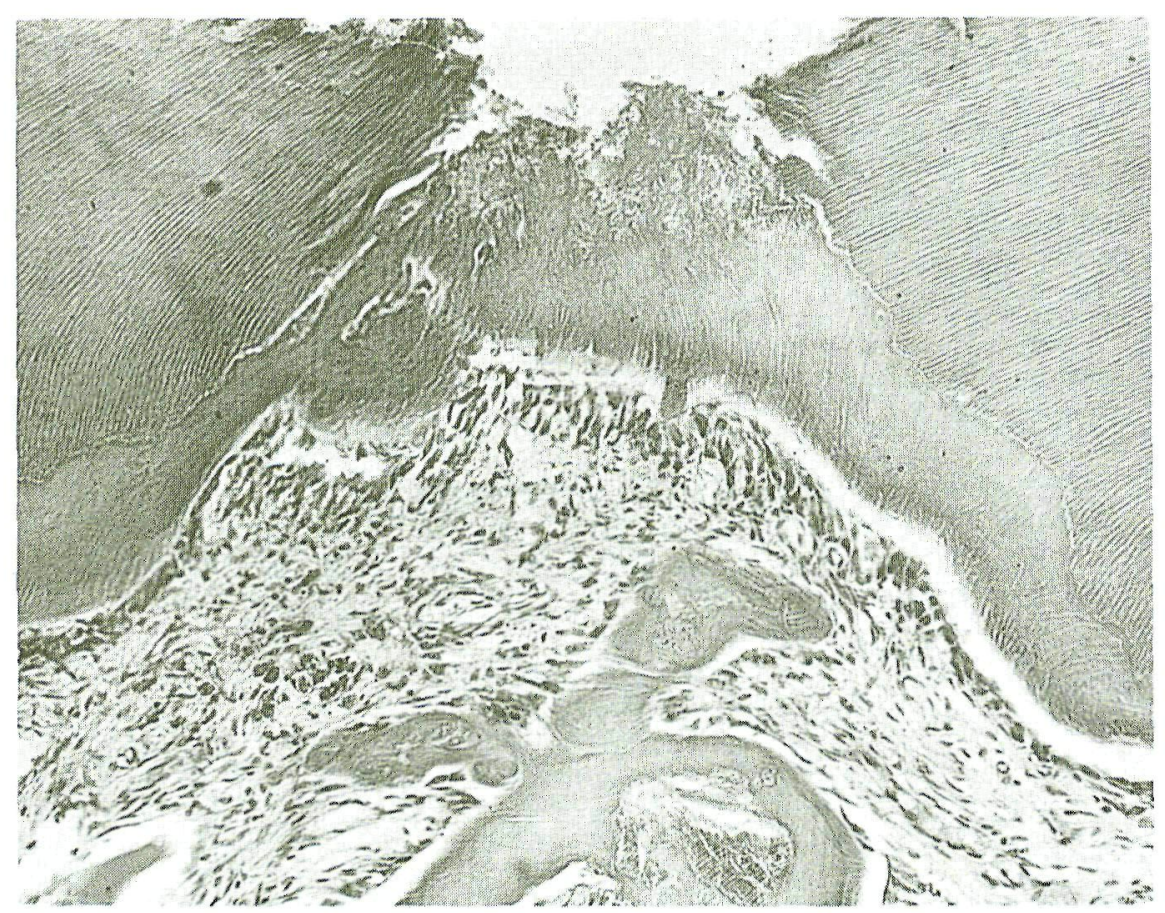

Fig. 3. This 5-week response to Dycal in a permanent tooth shows a reparative dentin bridge at the exposure site with the bridge composed of mostly atubular reparative dentin. The most pulpal area contains some tubules. Mag. $\times 64$.

permanent and primary teeth treated with MPC showed severe pulpal responses for the most part. No attempt at reparative dentin bridge formation was seen.

\section{Discussion}

The present study has shown differences between the calcium hydroxide-containing compounds tested. Dycal was observed to be the most successful agent in inducing reparative dentin bridge formation and subsequent healing of the dental pulp. An important point to emphasize is that the reparative dentin bridges observed in pulps treated with Dycal formed adjacent to the medicament interface and had no loss of pulp volume. This agrees with Stanley \&
Lundy (1972) and Tronstad (1974). Experimental MPC-12 was less successful than Dycal and slightly more successful than Pulpdent in stimulating reparative dentin bridge formation and subsequent pulp healing. Our results differ from those of Berk (1950) and Phaneuf et al. (1968) who reported that Pulpdent was very successful in pulp capping studies. In a histological comparative study Phaneuf showed Pulpdent stimulating reparative dentin after 28 days and after 50 days with Dycal. In this study Experimental MPC-12 and Pulpdent differed from Dycal in two respects; first, reparative dentin bridge formation was less frequent and, second, reparative dentin bridges that formed were at some distance from the exposure site, resulting in a loss of pulp 


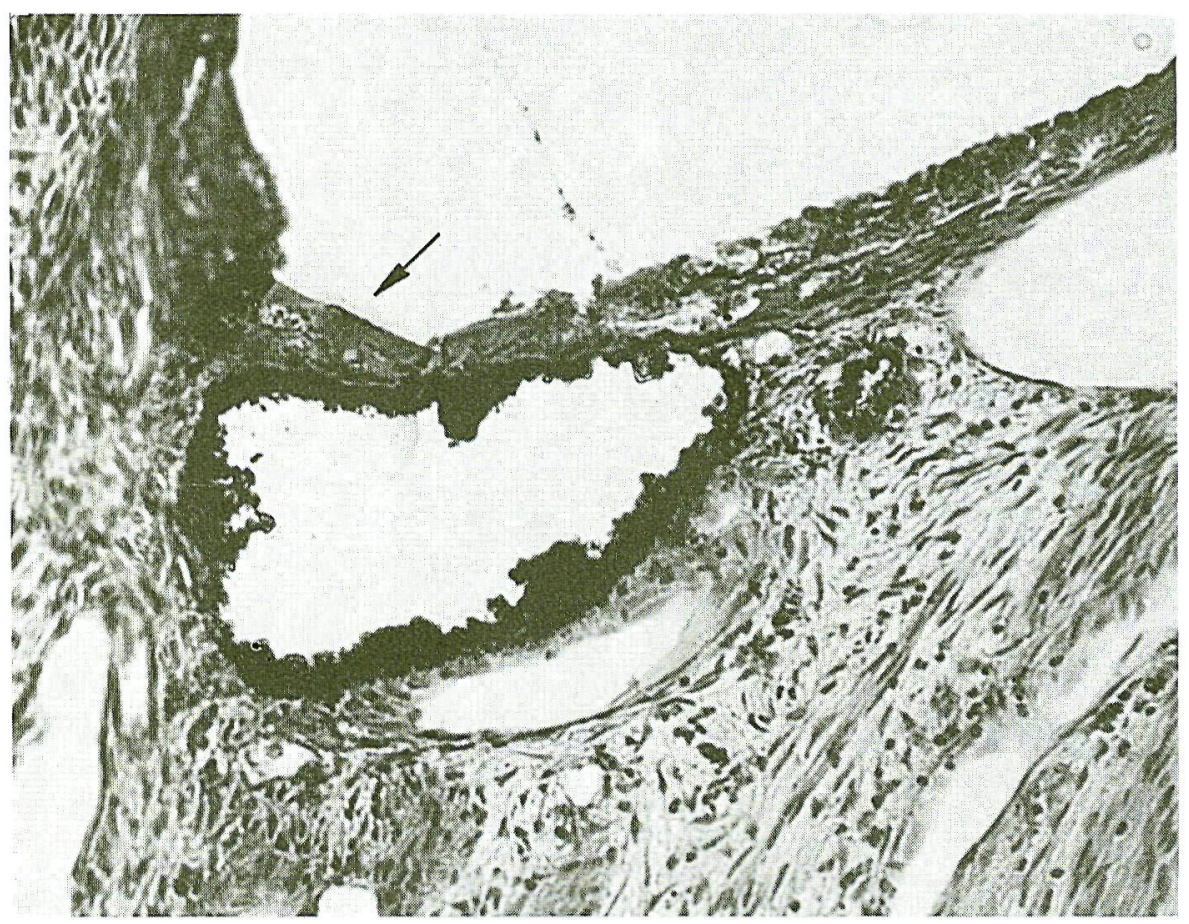

Fig. 4. A permanent tooth in which Experimental MPC-12 was placed. The arrow points to a zone of calcification adjacent to the medicament. The inflammation is moderate. The calcification is some distance apical to the exposure. Mag. $\times 160$.

volume. MPC was unable to initiate any reparative dentin bridge.

The histological responses of the pulpal tissue correlated with the ability of the medicaments to stimulate reparative dentin. In those teeth in which reparative dentin bridges formed the pulpal response was slight or slight to moderate. In those teeth where no dentin bridging was evident after 5 weeks the pulpal responses were generally moderate. After 5 weeks and by 8 weeks most responses were moderately severe to severe in pulps of permanent teeth with the exception of teeth treated with Dycal where the responses were moderate.

The responses of both primary and permanent teeth were quite similar. For example, a 3-day response to MPC in primary teeth and permanent teeth appeared to be similar in the number of inflammatory cells, the amount of tissue affected by the exposure and medicament placement, and the quantity of hemorrhage seen. The only differences noted could be attributed to the larger volume of pulp in the permanent teeth. The pulpal response was limited to a smaller area in permanent teeth as compared to the primary teeth. Having a larger percentage of the pulp involved may explain why the primary teeth responded less favorably.

The main purpose of this study was to see whether, by altering the $\mathrm{pH}$ of commercially available MPC ( $\mathrm{pH}$ of 10 ) to 12 , we could improve its ability to stimulate 


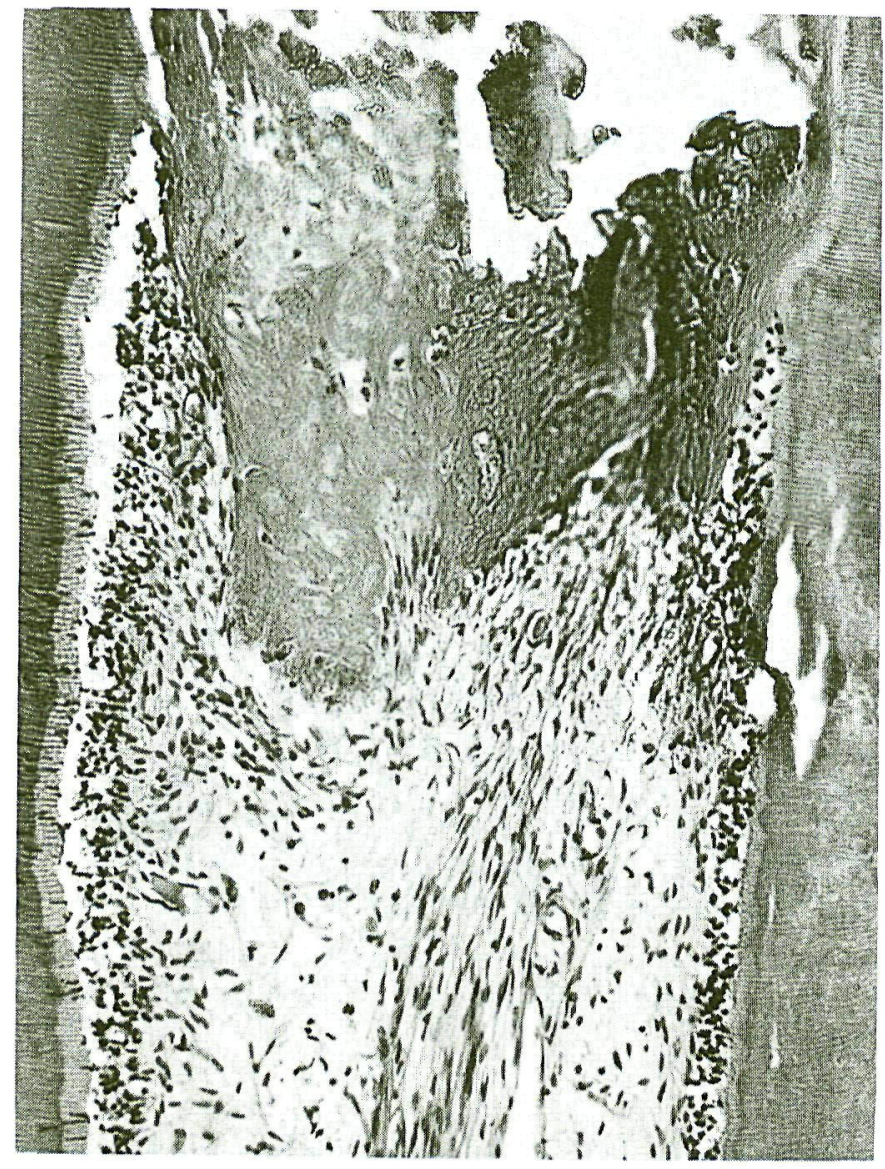

Fig. 5. A permanent tooth after 8 weeks in which Pulpdent has stimulated a nontubular reparative dentin bridge with a slight pulpal response. Bridging has occurred at a distance apical to the medicament interface. Mag. $\times 64$. reparative dentin. Other investigators (Fisher \& McCabe 1978, Tronstad \& Birkeland 1971) have placed the $\mathrm{pH}$ of Dycal at approximately 11 while Pulpdent has a pH of nearly 12. Results from our study showed that the $\mathrm{pH}$ is a factor in the success of a $\mathrm{Ca}(\mathrm{OH})_{2}$, but it is not the only factor. Experimental MPC-12 and Pulpdent were able to stimulate a dentin bridge only at some distance from the medicament interface. This was probably due to the amount of pulp tissue damage from these highly alkaline medicaments which in turn prevents tissue recovery and bridge formation at the medicament interface.
The 3-day pulpal responses of Experimental MPC-12 and Pulpdent showed tissue loss at some distance from the medicament interface, whereas less tissue damage occurred in relation to Dycal and MPC with less alkaline $\mathrm{pH}$ values. Stanley \& Lundy (1972) noted a zone of mummified and cauterized tissue beneath the Dycal at 11.days, whereas Tronstad (1974) stated that he observed vital tissue beneath the exposure and adjacent to the medicament. We found evidence of both types of response in our study and we feel that the difference between Stanley \& Lundy (1972) and Tronstad (1974) is one of semantics. We see some vital tissue 
adjacent to the medicament and this leads us to question the concept that necrosis is necessary for bridging to occur (Cotton 1974).

Studies done by Shubich et al. (1978) revealed that MPC allows very little egress of calcium ions from the set materials, whereas Dycal allowed more, and Pulpdent allowed considerably more than either. Fisher \& McCabe (1978) state that Hydrex (similar to MPC) has a paraffin matrix and is hydrophilic in nature. These characteristics may prevent diffusion of water into the set material, whereas the ethyl tolune sulfonamide of Dycal has polar groups which allow diffusion of water into the material resulting in a loss of the calcium ions from the set material. Since we tested two paraffin matrix type materials (MPC and Experimental MPC-12) with only a variation in the $\mathrm{pH}$, we feel that within this system changing the $\mathrm{pH}$ from 10 to 12 has improved the effectiveness of the material. Since the effectiveness is still not equal to Dycal, we conclude that the paraffin and not the $\mathrm{pH}$ may be the destructive factor. Although the optimal pH cannot be established in this study, the work of Fisher \& McCabe (1978) and Shubich et al. (1978) suggests that the release of calcium ions from the material might be more important than $\mathrm{pH}$ for the stimulation of pulp repair.

\section{References}

Berk, H. (1950) The effect of calcium hydroxidemethyl cellulose paste on the dental pulp. Journal of Dentistry for Children 17(4), 65-68.

Cotton, W. R. (1974) Bacterial contamination as a factor in healing of pulp exposures. Oral
Surgery, Oral Medicine, Oral Pathology 38(3), 441-450.

Cox, C. F., Heys, D. R. \& Heys, R. J. (1977) A gravity perfusion technique for lab animals. Laboratory Animals 6(4), 18-22.

Fisher, F. J. \& McCabe, J. F. (1978) Calcium hydroxide base materials. British Dental Journal 144(11), 341-344.

Mjör, I. A. \& Tronstad, A. M. (1972) Experimentally induced pulpitis. Oral Surgery, Oral Medicine, Oral Pathology 34(1), 102-108.

Nyborg, H. (1955) Healing process in the pulp on capping. A morphologic study. Experiment on surgical lesions of the pulp in dog and man. Acta Odontologica Scandinavica 13(Suppl.) 16.

Phaneuf, R. A., Frankl, S. N. \& Ruben, M. P. (1968) A comparable histological evaluation of three calcium hydroxide preparations on the human primary dental pulp. Journal of Dentistry for Children 35(1), 61-76.

Shubich, I., Miklos, F. L., Rapp, R. \& Draus, F. J. (1978) Release of calcium ions from pulpcapping materials. Journal of Endodontics 4(8), 242-244.

Stanley, H. R. \& Lundy, T. (1972) Dycal therapy for pulp exposures. Oral Surgery, Oral Medicine, Oral Pathology 35(5), 818-827.

Tronstad, L. (1974) Reaction of the exposed pulp to Dycal treatment. Oral Surgery, Oral Medicine, Oral Pathology 38(8), 945-953.

Tronstad, L. \& Birkeland, J. M. (1971) In vitro studies on the influence of cements on the alkaline effect of calcium hydroxide. Scandinavian Journal of Dental Research 79, 350-355.

Zander, H. A. (1939) Reaction of the pulp to calcium hydroxide. Journal of Dental Research 18, 373-379.

Zander, H. A. \& Glass, R. L. (1949) The healing of phenolized pulp exposures. Oral Surgery, Oral Medicine, Oral Pathology 2, 803-810.

\section{Address:}

Donald R. Heys, D.D.S., M.S.

The University of Michigan

5211 School of Dentistry

Ant Arbor, Michigan 48109

U.S.A. 
This document is a scanned copy of a printed document. No warranty is given about the accuracy of the copy. Users should refer to the original published version of the material. 